\title{
Impact of introducing the International Association of Diabetes and Pregnancy Study Groups (IADPSG) criteria on pregnancy outcomes in Japan
}

\author{
Ryosuke Shindo ${ }^{1)}$, Shigeru Aoki ${ }^{1)}$, Junko Kasai ${ }^{1)}$, Yusuke Saigusa ${ }^{2)}$, Sayuri Nakanishi ${ }^{1)}$ and Etsuko Miyagi ${ }^{3)}$ \\ 1) Perinatal Center for Maternity and Neonates, Yokohama City University Medical Center, Yokohama, Japan \\ 2) Department of Biostatistics, Yokohama City University Graduate School of Medicine, Yokohama, Japan \\ 3) Department of Obstetrics and Gynecology, Yokohama City University School of Medicine, Yokohama, Japan
}

\begin{abstract}
To estimate pregnancy complications in women newly diagnosed with gestational diabetes mellitus (GDM) according to the new International Association of Diabetes and Pregnancy Study Groups (IADPSG) criteria and verify the validity of introducing the IADPSG criteria in Japan. This retrospective study included data from women with singleton delivery at 22 weeks or later at a tertiary hospital during 2000-2009. We compared pregnancy outcomes between women who would now be diagnosed with GDM according to the IADPSG criteria but not by the old JSOG criteria (IGT group, $n=503$ ) and women with normal glucose tolerance according to both the criteria (NGT group, $n=2,789$ ). Multivariate analysis was performed and adjusted for background factors. Maternal age at delivery and pre-pregnancy BMI were significantly higher in the IGT group than in the NGT group, while gestational weeks at delivery did not differ between the groups. No difference was observed in the rates of GDM-related composite complications (defined as cases with at least one of the following: macrosomia, shoulder dystocia, neonatal hypoglycemia, neonatal hyperbilirubinemia, or neonatal respiratory distress syndrome) at $11.9 \%$ and $8.8 \%$ (adjusted odds ratio (OR) $1.30,95 \%$ confidence interval (CI) $0.90-1.87, p=0.16$ ). Pregnancy outcomes did not differ significantly between the IGT and NGT groups, except for frequencies of total neonatal admissions at $10.5 \%$ and $7.1 \%$, respectively (adjusted OR $1.55,95 \%$ CI $1.12-2.13, p<0.01$ ).
\end{abstract}

Key words: Gestational diabetes mellitus (GDM), International Association of the Diabetes and Pregnancy Study Group (IADPSG), Pregnancy, Japanese

BASED ON the results of the Hyperglycemia and Adverse Pregnancy Outcome (HAPO) study reported in 2008 [1], the International Association of Diabetes and Pregnancy Study Groups (IADPSG) defines the diagnosis of gestational diabetes mellitus (GDM) when a 75-g oral glucose tolerance test (OGTT) performed between 24 and 28 weeks of gestation exceeds one of the following: $92 \mathrm{mg} / \mathrm{dL}(5.1 \mathrm{mmol} / \mathrm{L})$ fasting plasma glucose (FPG), $180 \mathrm{mg} / \mathrm{dL}(10 \mathrm{mmol} / \mathrm{L})$ 1-hour post-glucose load, or $153 \mathrm{mg} / \mathrm{dL}(8.5 \mathrm{mmol} / \mathrm{L})$ 2-hour post-glucose load [2]. While many countries have adopted these international diagnostic criteria, the increase in the number of pregnant women diagnosed with GDM [3-9] has placed a heavy financial burden on medical care systems $[10,11]$.

Submitted Jul. 11, 2019; Accepted Aug. 7, 2019 as EJ19-0279

Released online in J-STAGE as advance publication Sep. 12, 2019 Correspondence to: Shigeru Aoki, Perinatal Center for Maternity and Neonate, Yokohama City University Medical Center, 4-57 Urafunecho, Minami-ku, Yokohama City, Kanagawa 232-0024, Japan.

E-mail: smyyaoki@yahoo.co.jp
Moreover, there is a lack of clear evidence that the new criteria reduce the incidence of perinatal adverse events. The American College of Obstetricians and Gynecologists (ACOG) does not support the IADPSG criteria [12].

Prior to the adoption of the IADPSG criteria, Japan had its own diagnostic criteria for GDM from the Japan Society of Obstetrics and Gynecology (JSOG), which required two or more of the following: fasting plasma glucose (FPG) concentration of $100 \mathrm{mg} / \mathrm{dL} \quad(5.5$ $\mathrm{mmol} / \mathrm{L})$, 1-h post-glucose load of $180 \mathrm{mg} / \mathrm{dL}$ (10 $\mathrm{mmol} / \mathrm{L}$ ), or 2-h post-glucose load exceeding $150 \mathrm{mg} / \mathrm{dL}$ $(8.3 \mathrm{mmol} / \mathrm{L})$ in a 75 -g OGTT. However, the old criteria were based on the National Diabetes Data Group (NDDG) criteria [13] and Carpenter and Coustan (C\&C) criteria [14], which means they were intended to predict the onset of postpartum type- 2 diabetes. It thus became evident that criteria aimed at preventing perinatal adverse events would be preferable. To achieve this, the diagnostic criteria used in Japan for GDM was changed in 2010 by introducing the IADPSG criteria [15]. As has 
been seen in other countries, the adoption of these criteria in Japan has led to a 2.7- to 5.7-fold increase in the prevalence of GDM. However, it remains unclear whether it has contributed to improving maternal and fetal prognoses [9, 16-19].

The objective of this study was to verify the validity of introducing the IADPSG criteria by examining pregnancy outcomes among women who were diagnosed with GDM based on the IADPSG criteria but not with the old JSOG criteria.

\section{Materials and Methods}

\section{Study design and subjects}

This single-center retrospective study used our center's database. Data were collected from an institutional perinatal database and the women's medical records. The study was approved by the ethics committee of Yokohama City University (No. B180700037).

The subjects were pregnant women who underwent a 75-g OGTT during pregnancy and had a singleton delivery at 22 weeks' gestation or later at the Perinatal Center for Maternity and Neonate of Yokohama City University Medical Center from January 1, 2000, to December 31, 2009. Following Japanese clinical guidelines [15], women considered to be at high risk of GDM undergo a 75-g OGTT at any time during pregnancy.

Basically, all pregnant women undergo a 2-step screening. Specifically, before 20 weeks of gestation, a 75-g OGTT is performed in women with certain risk factors (family history of diabetes, history of GDM, history of macrosomia [fetal weight $\geq 4,000 \mathrm{~g}$ ], history of shoulder dystocia, age $\geq 35$ years, obesity [pre-pregnant BMI $\geq 30]$ ) and with casual blood glucose concentration $\geq 95$ $\mathrm{mg} / \mathrm{dL}$. Then, all women except those with prepregnancy DM, overt DM; defined as FPG $\geq 126 \mathrm{mg} / \mathrm{dL}$ (7 $\mathrm{mmol} / \mathrm{L})$ ) or $\mathrm{HbA} 1 \mathrm{c} \geq 6.5 \%$, or already diagnosed with GDM in early pregnancy undergo universal screening with 50 -g glucose challenge test ( $50 \mathrm{~g} \mathrm{GCT}$ ) from 24 to 28 weeks' gestation. If the glucose challenge test result exceeds an hourly value of $140 \mathrm{mg} / \mathrm{dL}$, a 75-g OGTT is performed.

A 75-g OGTT is also performed in other cases, such as when urinary sugar level $\geq 2+$ is observed in a qualitative urine examination as part of a pregnancy health check, or in the presence of hydramnios or macrosomia (estimated fetal weight $\geq 4,000 \mathrm{~g}$ ).

Women with multiple pregnancies, pre-pregnancy DM, or overt DM were excluded. Finally, as subjects of the present study, we chose pregnant women treated as NGT before the introduction of the IADPSG criteria

We allotted the subjects into two groups. Women previously not diagnosed with GDM under the old JSOG criteria, but who received a diagnosis under the new IADPSG criteria, were placed in the impaired glucose tolerance (IGT) group, while those with normal glucose tolerance (NGT) under both the old JSOG and new IADPSG criteria were placed in the NGT group. Since these two groups had not been diagnosed with GDM under the old JSOG criteria at the time, neither received any intervention for GDM.

Maternal characteristics and pregnancy outcomes were compared between the groups. Data on the following characteristics was gathered from our department database and patient medical records: age (years), height $(\mathrm{cm})$, pre-pregnancy weight $(\mathrm{kg})$, pre-pregnancy body mass index (BMI) $\left(\mathrm{kg} / \mathrm{m}^{2}\right)$, weight at delivery $(\mathrm{kg})$, BMI at delivery $\left(\mathrm{kg} / \mathrm{m}^{2}\right)$, multiparity, gestational age at delivery (weeks), gestational weight gain (GWG) (kg), gestational age at GDM diagnosis (weeks).

The outcomes examined were: infant birth weight (g) and large-for-gestational-age (LGA), small-forgestational-age (SGA), macrosomia, shoulder dystocia, preeclampsia (PE), total cesarean deliveries, emergency caesarian deliveries, low Apgar score (APS), low umbilical arterial pH (UApH), neonatal hypoglycemia, neonatal hyperbilirubinemia, respiratory distress syndrome (RDS), neonatal intensive care unit (NICU) admission, total neonatal admissions, stillbirth, neonatal mortality within 1 week, and diabetes-related composite complications (at least one of the following: macrosomia, shoulder dystocia, neonatal hypoglycemia, neonatal hyperbilirubinemia, RDS).

Pre-pregnancy weight was the self-reported prepregnancy body weight at the initial examination. Babies were defined as LGA and SGA when their birth weights were above the 90th percentile and below the 10th percentile, respectively, for their gestation age. Macrosomia was defined as birth weight $\geq 4,000 \mathrm{~g}$. Shoulder dystocia was defined as when some kind of manipulation (McRoberts maneuver, suprapubic pressure, or intravaginal manipulation) was required to expulse the fetal shoulder. The definition of PE covered cases of hypertension (reproducible blood pressure $\geq 140 / 90 \mathrm{mmHg}$ ) and urinary protein creatinine ratio $>0.27$ between 20 weeks' gestation. Emergency cesarean delivery did not include scheduled caesarian delivery, even if it was advanced due to the onset of labor or rupture of membranes. Low APS was defined as Apgar score $<7$ at 5 minute and low UApH as $<7.1$. Neonatal hypoglycemia was defined as a plasma glucose level below $40 \mathrm{mg} / \mathrm{dL}$. Hyperbilirubinemia was defined as when phototherapy was required because infant birth weight- and age-based reference values were exceeded. RDS was defined by characteristic findings on chest radiographic examinations and requiring oxygen within $24 \mathrm{~h}$ after birth. NICU 
admission covered neonates who required intensive monitoring of their general condition. Total neonatal admission covered NICU admissions as well as cases that did not need intensive management but required interventions such as blood tests or phototherapy.

\section{Presentation of data and statistical analysis}

Statistical analyses were performed using JMP Pro version 12 (SAS Institute Inc., Cary, NC). Continuous variables were expressed as median (interquartile range) and categorical variables as frequencies and proportions. Comparison of the characteristics was performed with The Wilcoxon test for continuous variables and with the chi-square test were performed for categorical variables.

Multiple logistic regression analysis was performed for categorical variables to calculate adjusted odds ratios (ORs) and 95\% CI. Multiple linear regression analysis was performed for gestational age (weeks) at delivery and infant birth weight to calculate adjusted regression coefficients (RCs) and 95\% CI. These multivariate analyses were adjusted for maternal age, height, prepregnancy weight, and multiparity. Variables with $p<$ 0.05 were considered to be statistically significant.

\section{Results}

There was a total of 8,916 births during the study period. Among them, 3,772 women underwent 75-g OGTT during pregnancy. Of these, 286 women had multiple gestation and 22 women had FPG $\geq 126 \mathrm{mg} / \mathrm{dL}$, yielding a total of 305 women (some overlap) who were excluded. Further, 175 women who were diagnosed with GDM under the old JSOG criteria were excluded, leaving 3,292 subjects for the analysis (Fig. 1).

There were 503 women in the IGT group and 2,789 women in the NGT group. Table 1 shows the maternal characteristics of both groups. Maternal ages at delivery were 34 and 33 years, respectively, significantly higher in the IGT group $(p<0.01)$. Gestational ages at delivery did not differ between the groups $(p=0.07)$, though prepregnancy BMI and BMI at delivery were both significantly higher in the IGT group $(p<0.01, p<0.01)$. Gestational weight gain was $8.8 \mathrm{~kg}$ and $9.4 \mathrm{~kg}$, respectively, showing significantly less weight gain in the IGT group $(p<0.01)$

Table 2 shows the pregnant outcomes. No difference was observed in the rates of GDM-related composite complications at $11.9 \%$ and $8.8 \%$, respectively (adjusted OR $1.30,95 \%$ CI $0.90-1.89, p=0.16)$. Infant birth weight was 3,094 $\mathrm{g}$ and 3,038 $\mathrm{g}$, respectively, heavier in the IGT group but not significantly different (adjusted RC $10.0,95 \%$ CI $-14-34, p=0.41)$. Similarly, the frequencies of LGA did not differ significantly at $10.3 \%$

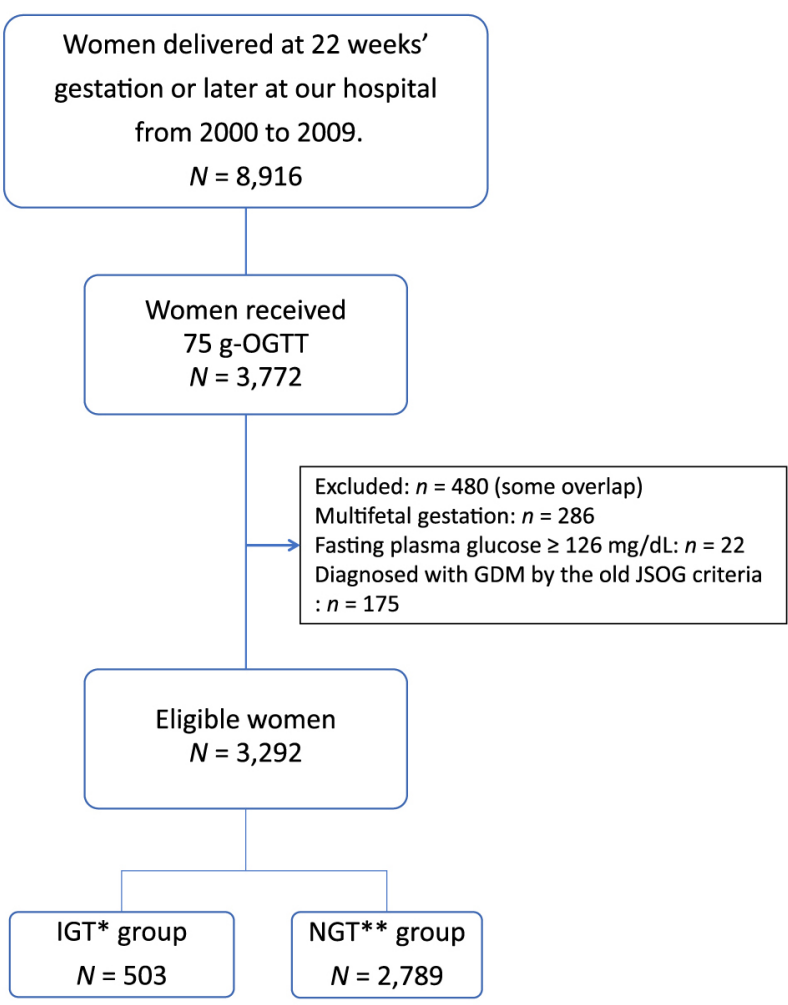

*IGT: impaired glucose tolerance women diagnosed with GDM by only the IADPSG criteria. **NGT: normal glucose tolerance

Fig. 1 Flow chart of subjects selection.

and $8.7 \%$, respectively (adjusted OR $1.11,95 \%$ CI $0.79-$ $1.53, p=0.53)$.

Rates of PE and caesarian delivery did not differ between the groups. The rate of NICU admission did not differ between the groups, however total neonatal admission, at $10.5 \%$ and $7.1 \%$, respectively, was significantly higher in the IGT group after adjustment (adjusted OR $1.55,95 \%$ CI $1.12-2.13, p<0.01)$.

\section{Discussion}

In the present study, we compared the mild IGT pregnant women with NGT pregnant women. The former had significantly higher frequencies of total neonatal admissions; however, there were no differences regarding other pregnancy outcomes between the two groups.

A 2016 secondary analysis of the HAPO study showed that pregnant women with mild IGT who were diagnosed with GDM under the IAGPSG criteria but not the C\&C criteria exhibited more frequent LGA, caesarian deliveries, neonatal hypoglycemia, and hyperbilirubinemia compared to pregnant women with NGT [20]. Further, a 2015 Canadian study found that pregnant women with mild IGT diagnosed under the IAGPSG criteria but not the old Canadian criteria exhibited more frequent hyper- 
Table 1 Maternal characteristics

\begin{tabular}{lccc}
\hline & $\begin{array}{c}\text { IGT group } \\
n=503\end{array}$ & $\begin{array}{c}\text { NGT group } \\
n=2,789\end{array}$ & $p$ value \\
\hline Age, y.o.: median (IQR) & $34(30-36)$ & $33(29-36)$ & $<0.01$ \\
Height, cm: median (IQR) & $158(155-162)$ & $158(155-162)$ & 0.26 \\
Prepregnancy weight, kg: median (IQR) & $54(50-62)$ & $52(48-58)$ & $<0.01$ \\
\hline Prepregnancy BMI, kg/m²: median (IQR) & $21.4(19.7-24.8)$ & $20.7(19.3-23.0)$ & $<0.01$ \\
Weight at delivery, kg: median (IQR) & $64.5(58.4-71.8)$ & $62.4(57.5-68.4)$ & $<0.01$ \\
BMI at delivery, kg/m²: median (IQR) & $25.3(23.3-28.2)$ & $24.9(23.0-27.2)$ & $<0.01$ \\
Gestational weight gain $(\mathrm{kg})$, median (IQR) & $8.8(6.2-11.9)$ & $9.4(7.1-12.0)$ & $<0.01$ \\
Multiparous, $n(\%)$ & $260(51.7 \%)$ & $1,319(47.3 \%)$ & 0.07 \\
\hline
\end{tabular}

IGT, Inpaired Glucose Tolerance; NGT, Normal Glucose Tolerance

Table 2 Pregnancy outcomes

\begin{tabular}{|c|c|c|c|c|c|c|}
\hline & $\begin{array}{l}\text { IGT group } \\
n=503\end{array}$ & $\begin{array}{c}\text { NGT group } \\
n=2,789\end{array}$ & $\begin{array}{l}\text { crude OR } \\
(95 \% \mathrm{CI})\end{array}$ & $p$ value & $\begin{array}{c}\text { adjusted }{ }^{* 2} \mathrm{OR} \\
(95 \% \mathrm{CI})\end{array}$ & $p$ value \\
\hline Diabetes related composite complications*1, $n(\%)$ & $60(11.9)$ & $246(8.8)$ & $1.40(1.04-1.89)$ & 0.03 & $1.30(0.90-1.87)$ & 0.16 \\
\hline Macrosomia, $n(\%)$ & $7(1.4)$ & $39(1.4)$ & $1.00(0.44-2.24)$ & 0.99 & $0.80(0.32-1.71)$ & 0.58 \\
\hline Shoulder dystocia, $n$ (\%) & $8(1.6)$ & $36(1.3)$ & $1.24(0.57-2.67)$ & 0.59 & $1.08(0.46-2.26)$ & 0.84 \\
\hline Neonatal hypoglycemia, $n(\%)$ & $7(1.4)$ & $21(0.8)$ & $1.86(0.79-4.40)$ & 0.15 & $1.84(0.71-4.20)$ & 0.19 \\
\hline Neonatal hyperbililbiremia, $n$ (\%) & $37(7.4)$ & $144(5.2)$ & $1.46(1.00-2.12)$ & $<0.05$ & $1.48(0.98-2.10)$ & 0.06 \\
\hline RDS, $n(\%)$ & $6(1.2)$ & $28(1.0)$ & $1.19(0.49-2.89)$ & 0.7 & $1.14(0.42-2.62)$ & 0.78 \\
\hline Pre-eclampsia, $n(\%)$ & $10(2.0)$ & $50(1.8)$ & $1.11(0.56-2.21)$ & 0.76 & $0.89(0.42-1.71)$ & 0.73 \\
\hline LGA, $n(\%)$ & $52(10.3)$ & $243(8.7)$ & $1.21(0.88-1.66)$ & 0.24 & $1.11(0.79-1.53)$ & 0.53 \\
\hline $\mathrm{SGA}, n(\%)$ & $40(8.0)$ & $266(9.5)$ & $0.82(0.58-1.16)$ & 0.26 & $0.85(0.59-1.20)$ & 0.36 \\
\hline Low APS, $n(\%)$ & $39(7.8)$ & $195(7.0)$ & $1.12(0.78-1.60)$ & 0.54 & $1.14(0.78-1.62)$ & 0.49 \\
\hline Low UApH, $n(\%)$ & $13(2.6)$ & $36(1.3)$ & $2.03(1.07-3.85)$ & 0.03 & $2.00(1.00-3.75)$ & 0.05 \\
\hline Emergency CS, $n(\%)$ & $58(11.5)$ & $291(10.4)$ & $1.12(0.83-2.01)$ & 0.27 & $1.03(0.75-1.39)$ & 0.87 \\
\hline Total CS, $n(\%)$ & $138(27.4)$ & $662(23.7)$ & $1.21(0.98-1.50)$ & 0.08 & $1.06(0.85-1.33)$ & 0.58 \\
\hline NICU admission, $n(\%)$ & $25(5.0)$ & $109(3.9)$ & $1.29(0.82-2.00)$ & 0.27 & $1.32(0.83-2.04)$ & 0.24 \\
\hline Neonatal admission (include NICU), $n(\%)$ & $53(10.5)$ & $198(7.1)$ & $1.54(1.12-2.12)$ & $<0.01$ & $1.55(1.12-2.13)$ & $<0.01$ \\
\hline \multirow[t]{2}{*}{ Stillbirth, $n(\%)$} & $1(0.2)$ & $7(0.3)$ & $0.79(0.10-6.45)$ & 0.83 & $0.98(0.05-5.60)$ & 0.98 \\
\hline & & & $\begin{array}{l}\text { crude RC } \\
(95 \% \mathrm{CI})\end{array}$ & $p$ value & $\begin{array}{c}\text { adjusted }{ }^{* 3} \mathrm{RC} \\
(95 \% \mathrm{CI})\end{array}$ & $p$ value \\
\hline Neonatal death (<1 week), $n$ (\%) & 0 & $1(0.04)$ & - & - & - & - \\
\hline Gestational weeks at delivery (weeks), medean (IQR) & $39.4(38.3-40.3)$ & $39.4(37.6-40.3)$ & $-0.06(-0.16-0.03)$ & 0.19 & $-0.05(-0.15-0.04)$ & 0.27 \\
\hline Infant birth weight (g), medean (IQR) & $3,094(2,766-3,380)$ & $3,038(2,768-3,320)$ & $19.01(-5.2-43.2)$ & 0.12 & $10.0(-14.0-34.1)$ & 0.41 \\
\hline
\end{tabular}

OR, Odds ratio; RC, Regression Coefficient; IQR, Interquatile range; IGT, impaired glucose tolerance; NGT, normal glucose tolerance

*1 diabetes-related composite complications defined as cases with at least one of the following: macrosomia, shoulder dystocia, neonatal hypoglycemia, neonatal hyperbilirubinemia, or RDS.

*2 Adjusted Odds Ratioadjusted for NGT group, adjusted for maternal age, height, pre-pregnancy weight, multi paraous

*3 Adjusted Regression Coefficient for NGT group, adjusted for maternal age, height, pre-pregnancy weight, multi paraous

tensive disorder of pregnancy (HDP), caesarian delivery, and LGA, and higher birth weights than pregnant women with NGT [21]. A 2017 Australian study reported higher rates of macrosomia/LGA, nursery admission, labor intervention, HDP, and other issues among pregnant women with mild IGT diagnosed under the IAGPSG criteria but not the old Australian criteria, compared to pregnant women with NGT [22]. This shows that preg- 
nant women who were not diagnosed under their nations' old criteria but were diagnosed with mild IGT under the IADPSG criteria are at higher risk than women with NGT. The present study did not observe an increase in perinatal adverse events that previous research has shown except total neonatal admission. One possible reason for this is that the timing for performing 75-g OGTT in Japan does not match the IADPSG recommendation. The JSOG places no limits on the gestational age when a 75-g OGTT can be performed on pregnant women suspected of having IGT [15], meaning that many women are tested during a period other than 24-28 weeks gestation. In fact, about $60 \%(1,991 / 3,292)$ of the subjects had received 75-g OGTT outside the range of 24 to 28 weeks. In previous research into issues unique to Japan, we did not observe an improvement in perinatal adverse events in a group that was diagnosed with GDM before 20 weeks' gestation and received interventions early [23], though this result could have been influenced by the fact that many of the subjects were diagnosed at a time not recommended by the IADPSG criteria. That said, the present study also found an increase in neonatal admissions, which suggests that the introduction of the IADPSG criteria has enabled the detection of cases of mild IGT.

In this study, we did not examine GDM morbidity rates before and after introducing the IADPSG criteria, although some previous Japanese studies have reported 2.7-5.7-fold increases [9, 16-19]. The cost-effectiveness of introducing the IADPSG criteria has been studied in other countries $[10,11]$, but remains controversial. This is because the introduction of these criteria would lead to an increase in the number of patients diagnosed with GDM, which would in turn cause an increase in overall medical costs. However, if a substantial improvement in pregnancy and delivery outcomes can be achieved, then the introduction of these criteria can be considered appropriate and the drawbacks can be ignored. There is a need for further investigations to examine this topic from a cost-benefit standpoint.

This study had the following limitations. First, it was a single-center, retrospective study of a small number of cases. Second, treatment of GDM has two aims, to improve the perinatal outcome of the index pregnancy and to improve the long-term health of both the mother and the offspring. However, the latter was not examined in the present study.

In conclusion, pregnant women with IGT, who were within the normal range under the old JSOG criteria but to be newly diagnosed under the IADPSG criteria, were at higher risk of neonatal admission than were pregnant women with NGT; however, there were no differences in other pregnancy outcomes between the two groups. We recommend that the appropriateness of introducing the IADPSG criteria in Japan should be reassessed, including at the timing of 75-g OGTT.

\section{Acknowledgments}

None.

\section{Declaration of Interest}

None.

\section{References}

1. Metzger BE, Lowe LP, Dyer AR, Trimble ER, Chaovarindr U, et al. (2008) Hyperglycemia and adverse pregnancy outcomes. N Engl J Med 358: 1991-2002.

2. Metzger BE, Gabbe SG, Persson B, Buchanan TA, Catalano PA, et al. (2010) International association of diabetes and pregnancy study groups recommendations on the diagnosis and classification of hyperglycemia in pregnancy. Diabetes Care 33: 676-682.

3. Shang M, Lin L (2014) IADPSG criteria for diagnosing gestational diabetes mellitus and predicting adverse pregnancy outcomes. J Perinatol 34: 100-104.

4. Ethridge JK Jr, Catalano PM, Waters TP (2014) Perinatal outcomes associated with the diagnosis of gestational diabetes made by the international association of the diabetes and pregnancy study groups criteria. Obstet Gynecol 124: 571-578.

5. Ekeroma AJ, Chandran GS, McCowan L, Ansell D, Eagleton C, et al. (2015) Impact of using the International
Association of Diabetes and Pregnancy Study Groups criteria in South Auckland: prevalence, interventions and outcomes. Aust N Z J Obst Gynaecol 55: 34-41.

6. Duran A, Saenz S, Torrejon MJ, Bordiú E, Del Valle L, et al. (2014) Introduction of IADPSG criteria for the screening and diagnosis of gestational diabetes mellitus results in improved pregnancy outcomes at a lower cost in a large cohort of pregnant women: the St. Carlos Gestational Diabetes Study. Diabetes Care 37: 2442-2450.

7. Bodmer-Roy S, Morin L, Cousineau J, Rey E (2012) Pregnancy outcomes in women with and without gestational diabetes mellitus according to the International Association of the Diabetes and Pregnancy Study Groups criteria. Obstet Gynecol 120: 746-752.

8. Moses RG, Morris GJ, Petocz P, San Gil F, Garg D (2011) The impact of potential new diagnostic criteria on the prevalence of gestational diabetes mellitus in Australia. Med J Aust 194: 338-340. 
9. Kodama Y, Sameshima H, Ohashi M, Ikenoue T (2013) Impact of new gestational diabetes mellitus criteria on stillbirth: a regional population-based study in Japan. $J$ Obstet Gynaecol Res 39: 1242-1245.

10. Round JA, Jacklin P, Fraser RB, Hughes RG, Mugglestone MA, et al. (2011) Screening for gestational diabetes mellitus: cost-utility of different screening strategies based on a woman's individual risk of disease. Diabetologia 54: 256-263.

11. Flack JR, Ross GP, Ho S, McElduff A (2010) Recommended changes to diagnostic criteria for gestational diabetes: impact on workload. Aust N Z J Obstet Gynaecol 50: 439443.

12. Committee on Practice Bulletins-Obstetrics (2018) ACOG practice bulletin No. 190: gestational diabetes mellitus. Obstet Gynecol 131: e49-e64.

13. National Diabetes Data Group (1979) Classification and diagnosis of diabetes mellitus and other categories of glucose intolerance. Diabetes 28: 1039-1057.

14. Carpenter MW, Coustan DR (1982) Criteria for screening tests for gestational diabetes. Am J Obstet Gynecol 144: 768-773.

15. Minakami H, Hiramatsu Y, Koresawa M, Fujii T, Hamada $\mathrm{H}$, et al. (2011) Guidelines for obstetrical practice in Japan: Japan Society of Obstetrics and Gynecology (JSOG) and Japan Association of Obstetricians and Gynecologists (JAOG) 2011 edition. J Obstet Gynaecol Res 37: 1174-1197.

16. Morikawa M, Yamada T, Yamada T, Akaishi R, Nishida $\mathrm{R}$, et al. (2010) Change in the number of patients after the adoption of IADPSG criteria for hyperglycemia during pregnancy in Japanese women. Diabetes Res Clin Pract 90: 339-342.

17. Okada T, Iwashina M, Kasatani T, Kanno H, Yoshie M, et al. (2013) Clinical outcomes of pregnancies complicated with and treated for gestational diabetes mellitus: consequences of screening under the IADPSG criteria. Diabetol Int 4: 186-189.

18. Fukatsu M, Takai Y, Matsunaga S, Era S, Ono Y, et al. (2017) Diagnosis and potential management of gestational diabetes mellitus using the international association of diabetes and pregnancy study groups criteria. J Obstet Gynaecol Res 43: 272-280.

19. Ikenoue S, Miyakoshi K, Saisho Y, Sakai K, Kasuga Y, et al. (2014) Clinical impact of women with gestational diabetes mellitus by the new consensus criteria: two year experience in a single institution in Japan. Endocr $J$ 61: 353-358.

20. Waters TP, Dyer AR, Scholtens DM, Dooley SL, Herer E, et al. (2016) Maternal and neonatal morbidity for women who would be added to the diagnosis of GDM using IADPSG criteria: a secondary analysis of the Hyperglycemia and Adverse Pregnancy Outcome Study. Diabetes Care 39: 2204-2210.

21. Mayo K, Melamed N, Vandenberghe H, Berger H (2015) The impact of adoption of the international association of diabetes in pregnancy study group criteria for the screening and diagnosis of gestational diabetes. Am J Obstet Gynecol 212: 224. e1-e9.

22. Tan HLE, Luu J, Caswell A, Holliday E, Attia J, et al. (2017) Impact of new International Association of Diabetes and Pregnancy Study Groups (IADPSG) diagnostic criteria on perinatal outcomes in a regional tertiary hospital in New South Wales, Australia. Diabetes Res Clin Pract 134: 191-198.

23. Hagiwara Y, Kasai J, Nakanishi S, Saigusa Y, Miyagi E, et al. (2018) Should the IADPSG criteria be applied when diagnosing early-onset gestational diabetes? Diabetes Res Clin Pract 140: 154-161. 